96 Curtius: Nomenclatur von organ. Verbindungen etc.

\title{
Mittheilungen aus dem chemischen Institut der Universität Kiel.
}

\section{Nomenclatur vou organisehen Verbindungen, welche zwei unter sich gebundene Stickstoffatome enthalten; \\ von}

Th. Curtius.

Die Entdeckung des Diamids hat in wenigen Jahren die Auffindung einer grossen Anzahl von Verbindungen, welche zwei unter sich gebundene Stickstoffatome enthalten, zur Folge gehabt, eine Anzahl, welche in Zukunft in dem Maasse wachsen wird, in welchem es gelingt sich mit den mannigfaltigen, merkwürdigen Eigenschaften dieses Stickstoffwasserstoffs völlig vertraut $\mathrm{zu}$ machen. Eine einigermassen einheitliche Nomenclatur für die Hydrazinderivate, wie für ihre nächsten Verwandten. die fetten Diazoverbindungen, festzustellen, erscheint daher dringend geboten. Ich werde mich nun in demnächst und später zu veröffentlichenden Abhandlungen einer Bezeichnungsweise dieser Substanzen bedienen, welche nach folgenden wenigen, sehr einfachen und zum Theil schon längst bewährten Gesichtspunkten durchgeführt werden wird. Diese Bezeichnungsweise kann sich allerdings nicht auf die zahlreichen ringförmigen Abkömmlinge des Diamids erstrecken, welche aus mehreren Kohlenstoffatomen, ausser den beiden Stickstoffatomen, gebildet sind, z. B. auf die Pyrazolone. Bei der Benennung derartiger Körper wird man sich leider auch in der Zukunft willkürlich, so gut es geht, behelfen müssen. Ferner wird diese Nomenclatur zunächst nur bei solchen Derivaten angewandt werden, in welchen das Stickstoffdoppelatom nicht unmittelbar mit Benzolresten zusammenhängt, obwohl die Glieder der grossen Klasse der aromatischen Azo- und Hydrazinverbindungen sich ebenfalls zwanglos nach demselben Princip benennen lassen.

Die Bezeichnungsweise von organischen Verbindungen, welche zwei unter sich gebundene Stickstoffatome enthalten, beruht darauf, dass man Folgendes consequent beachtet:

1. Zwei unter sich einfach gebundene Stickstoffatome, 
welche das vierwerthige Radical $\left(\begin{array}{l}N \\ \vdots\end{array}\right)^{\prime \prime \prime \prime}$ repräsentiren, bilden die "Azigruppe“ (am Ende eines Wortes "azin" zu schreiben).

2. Zwei unter sich doppelt gebundene Stickstoffatome, das zweiwerthige Radical $\left(\begin{array}{l}\mathbf{N} \\ \mathbf{N}\end{array}\right)^{\prime \prime}$ vorstellend, bilden die altbekannte "Azogruppe" (am Ende eines Wortes „azon" zu schreiben).

3. Sobald eines dieser Radicale partiell oder ganz mit Wasserstoff gesättigt wird, tritt vor die Worte "A $\mathrm{zi}^{\text {" }}$ und "A zo" die Silbe „Hydr-“.

So entstehen die Bezeichnungen „Hydrazo“, „Hydrazi“ (am Ende eines Wortes „Hydrazon“, „Hydrazin" zu schreiben).

Sämmtliche, überhaupt nur mögliche Körper mit zwei unter einander gebundenen Stickstoffatomen, es gehören dahin alle bekannten Azo-, Diazo-, Bydrazinverbindungen, leiten sich wenn man diese wenigen Gesichtspunkte im Auge behält, vom Hydrazon (Diimid, noch nicht dargestellt), oder vom Hydrazin (Diamid) durch Ersatz von. Wasserstoff ab.

$\begin{array}{cc}\mathrm{NH} & \mathrm{NH}_{2} \\ \| & \mathrm{NH}_{2} \\ \text { Hydrazon } & \text { Hydrazin } \\ \text { (Diimid) } & \text { (Diamid) }\end{array}$

Zwanglos ergeben sich zunächst so die Benennungen für die zahlreichen Hydrazinderivate, welche durch Einwirkung von Hydrazinhydrat auf Säureester, Säureamide, Säurechloride oder Glycolsäureester entstehen, welche primäre oder symm. secundäre Hydrazine repräsentiren:

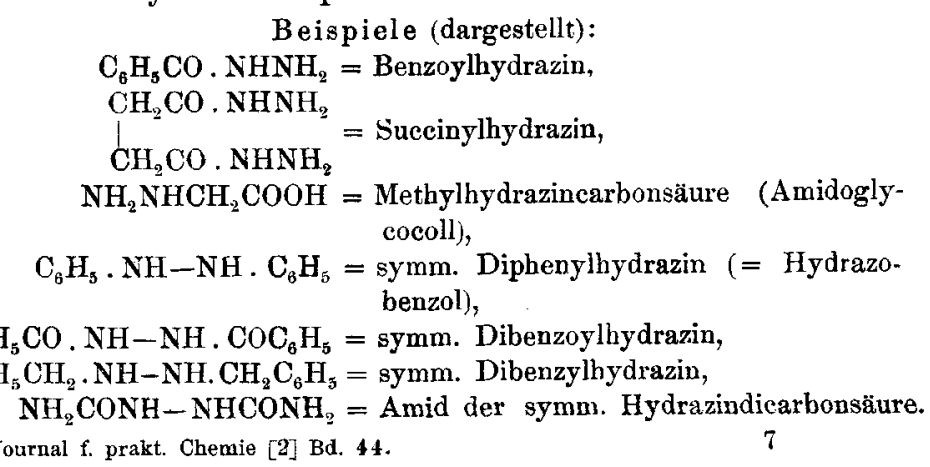


98 Curtius: Nomenclatur von organ. Verbindungen etc.

Ferner für die schwierig zu erhaltenden Einwirkungsprodukte von Aldehyden und gewöhnlichen Monoketonen auf überschüssiges Hydrazinhydrat, welche als unsymm. secundäre Hydrazine aufzufassen sind:

Beispiele (dargestellt):

$\mathrm{C}_{6} \mathrm{H}_{5} \mathrm{CH}: \mathrm{N}-\mathrm{NH}_{2}=$ Benzalhydrazin,

$\left(\mathrm{C}_{6} \mathrm{H}_{\vec{b}}\right)_{2} \mathrm{C}: \mathrm{N}-\mathrm{NH}_{2}=$ Diphenylmethylenhydrazin.

Weiter für die zahlreich gewonnenen tertiären Hydrazine, welche aus den primären durch Condensation mit Aldehyden oder Ketonen sich bilden :

Beispiele (dargestellt):

$\mathrm{C}_{6} \mathrm{H}_{5} \mathrm{CO}$. NHN : $\mathrm{CHC}_{6} \mathrm{H}_{5}=$ Benzoylbenzalhydrazin,

$\mathrm{C}_{6} \mathrm{H}_{5} \mathrm{CO}$. NHN : $\mathrm{C}\left(\mathrm{CH}_{3}\right)_{2}=$ Benzoyldimethylmethylenhydrazin ete.

Endlich für die grosse Klasse der Azine, welche nicht nur aus den unsymm. secundären Hydrazinen durch Einwirkung eines zweiten Moleküles Aldehyd oder Keton entstehen, sondern sich überhaupt mit Vorliebe durch Einwirkung von Hydrazinhydrat auf Aldehyde und Ketone bilden:

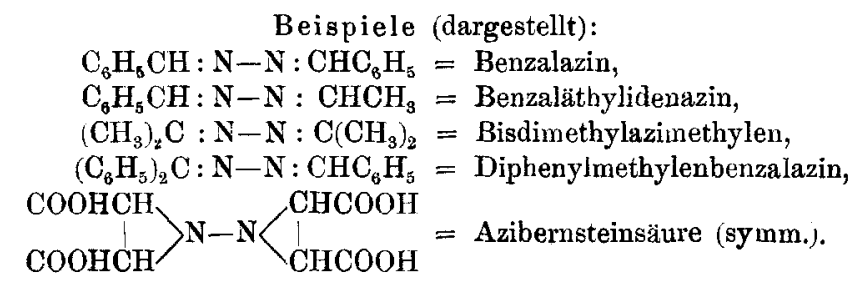

Aus nachfolgenden, ebenfalls aus den oben aufgestellten Grundsätzen abgeleiteten Principien ergiebt sich nun weiter auch eine einfache und übersichtliche Bezeichnungsweise für die fetten, sogenannten Diazoverbindungen und die aus ihnen durch Reduction darstellbaren Hydrazine, in welchen allen die "Azogruppe"i $\left(\begin{array}{l}\mathrm{N} \\ 1 \\ \mathrm{~N}\end{array}\right)^{\prime \prime}$ oder die „Azigruppe“ $\left(\begin{array}{l}\mathbf{N} \\ \mathbf{N}\end{array}\right)^{\prime \prime \prime \prime}$, resp. die symm. „Hydrazingruppe“ $\left(\begin{array}{c}\mathrm{NH} \\ \mathrm{NH}\end{array}\right)^{\prime}$ mit einem und demselben Kohlenstoffatom verbunden ist.

Durch den Eintritt dieser Radicale an Stelle von Wasserstoff in ein Molekül Methan oder ein Molekül Ammoniak ent- 
stehen nämlich folgende vier dreigliedrige Ringe, von denen bis heute allerdings nur einer in freiem Zustande dargestellt worden ist, von denen allen aber zahlreiche Derivate bekannt sind :<smiles></smiles>

Azomethylen

(unbekannt) (trimolekular bekannt als Kern der

Tris-Azoessigsäure)<smiles>C1CCNC1</smiles>

Hydrazimethylen (unbekannt)

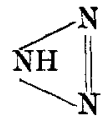

Azoimid (bekannt)

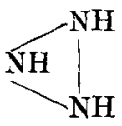

Hydraziimid (noch nicht isolirt)

Die gegebene Bezeichnungsweise für die 4 Körper ist vollkommen scharf, wenn man die früher mitgetheilten drei Gesichtspunkte im Auge behält. Es ist aber zu bemerken, dass man beispielsweise die Verbindung $\mathrm{CH}_{2}^{\mathrm{NH}}$ nicht „Hydrazomethan" statt Hydrazomethylen benennen darf, da ein Körper von der Zusammensetzung $\mathrm{C}(\mathrm{NH})_{2}$ ebenfalls als „Hydrazomethan" zu bezeichnen wäre. Ebenso ist ein "Azomethan" von der Zusammensetzung $\mathrm{C}_{\mathrm{N}_{2}} \mathrm{~N}_{2}$ denkbar. Auch das Azoimid darf man nicht als Azoammoniak bezeichnen, da einer solchen Benennung auch die Zusammensetzung der unbekannten Stickstoffwasserstoffverbindung $\mathrm{NH}_{2}-\mathrm{N}=\mathrm{N}-\mathrm{NH}_{2}$ entspricht. Man würde heute den zuletzt genannten Unterscheidungen noch keinen besonderen Werth beizulegen brauchen, da die meisten dieser stickstoffreichen Verbindungen noch hypothetischer Natur sind, wenn nicht die nousten Erfahrungen über das Wesen des Stickstoffs darauf hindeuteten, dass die Existenz solcher Verbindungen keineswegs mehr in das Gebiet des Unwahrscheinlichen gehört.

Von dem Azoimid, dem Azomethylen, Hydrazimethylen und Azimethylen (das Hydraziimid kommt vorläufig noch nicht in Betracht) lassen sich nun alle bisher dargestellten fetten sogenannten Diazoverbindungen und ibre Reductionsprodukte, welche Hydrazinverbindungen sind, einheitlich ableiten. 
100 Curtius: Nomenclatur von organ. Verbindungen etc.

Beispiele (sämmtliche bekannt):
I. Derivate des
Azomethylens

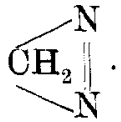

A) mit Alkylen und Acidylen:

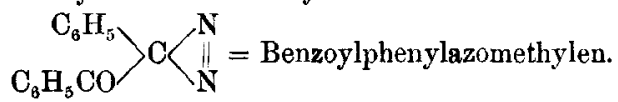

B) mit Alkylen und Carboxylen:<smiles>CCOC(=O)C1CN=N1</smiles>

$=$ Azomethylencarbonsäureester

(Diazoessigester).
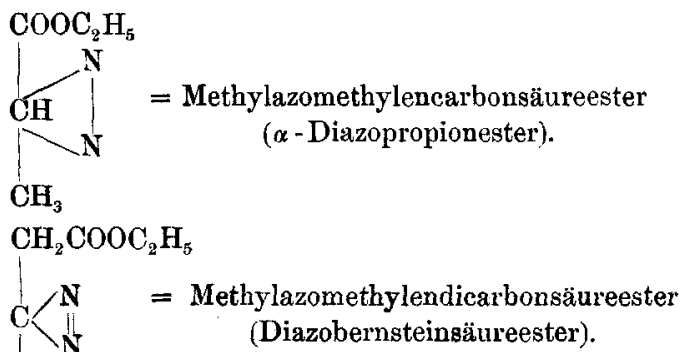
$\mathrm{COOC}_{2} \mathrm{H}_{3}$

II. Derivate des Hydrazimethylens $\mathrm{CH}_{2}$

A) mit Alkylen und Acidylen:
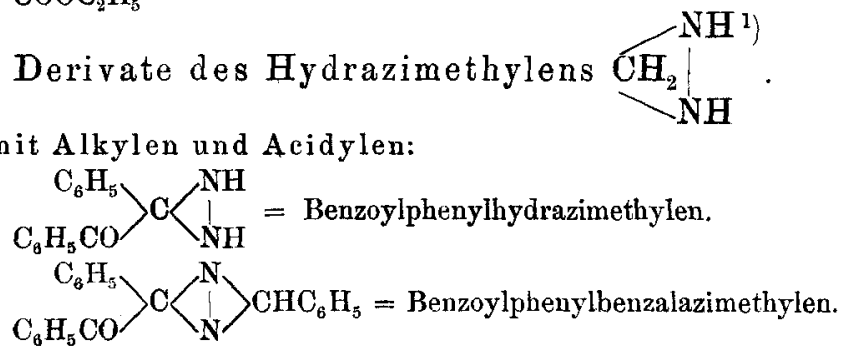

1) „Hydrazomethylen" statt „Hydrazimethylen" zu sagen, ist zwar inconsequent, da bei ersterem keine doppelte Bindung zwischen den Stickstoffatomen vorhanden ist, aber ohne sonderliche Verwirrung hervorzurufen, möglich, da „Hydrazo“, resp. „Hydrazon“ als solches nur die gesättigte Verbindung NH $_{\mathbf{N}}^{\mathrm{NH}}$, das hypothetische Diimid, kein Radical mehr bedeuten kann. Nach vorstehender Nomenclatur ist das altbekannte "Hydrazobenzol" $\mathrm{C}_{6} \mathrm{H}_{5}-\mathrm{NH}-\mathrm{NHC}_{6} \mathrm{H}_{3}$ als symm. Diphenylhydrazin zu bezeichnen, während der Name „Hydrazobenzol", resp. „Phenylhydrazon" für den unbekannten Körper $\mathrm{C}_{6} \mathrm{H}_{5} . \mathrm{N}: \mathrm{NH}$ zu reserviren bleibt. 
Curtins: Ueb. Diammoniumsemisulfat $\left(\mathrm{N}_{2} \mathrm{H}_{4}\right)_{2} \cdot \mathrm{H}_{2} \mathrm{SO}_{4} \cdot 101$

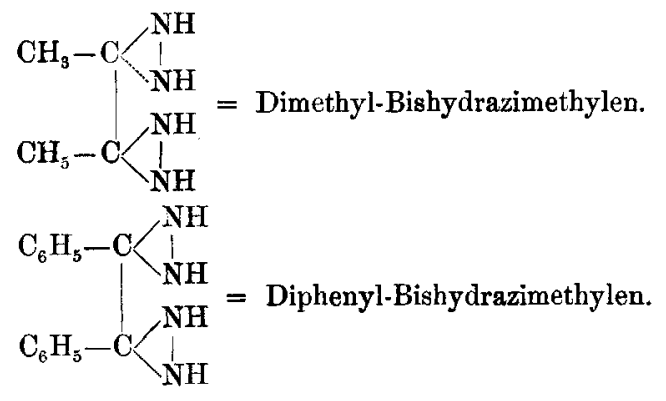

B) mit Alkylen und Carboxylen:

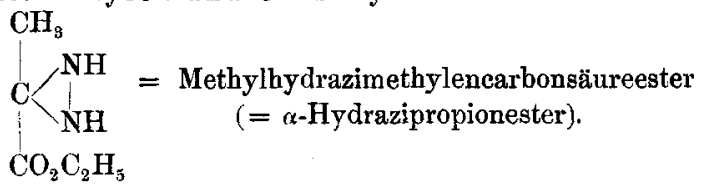

III. Derivate des Azoimids.

A) mit Alkylen:

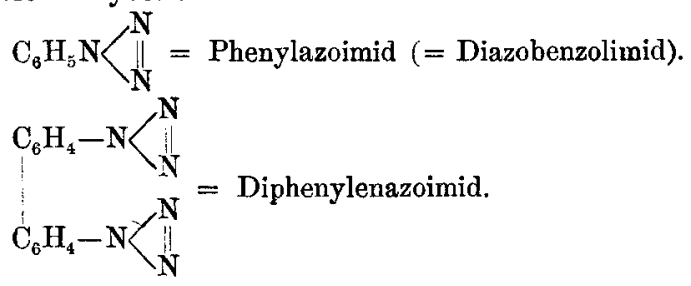

B) mit Acidylen:

$\left.\mathrm{C}_{6} \mathrm{H}_{5} \mathrm{CON}\right\rangle_{\mathrm{N}}^{\mathrm{N}}=$ Benzoylazoimid.
$\mathrm{C}_{6} \mathrm{H}_{5} \mathrm{NH} . \mathrm{CH}_{2} \mathrm{CO} \angle_{\mathrm{N}}^{\mathrm{N}}=$ Phenylamidoacetylazoimid.

Kiel, im Mai 1891.

\section{Ueber Diammoninmsemisulfat $\left(\mathrm{N}_{2} \mathrm{H}_{4}\right)_{2} \cdot \mathrm{H}_{2} \mathrm{SO}_{4}$; von}

Th. Curtius.

In der von $\mathrm{Schulz}$ und mir $^{1}$ ) veröffentlichten Abhandlung über „Hydrazinhydrat und die Halogenverbindúngen des Diammoniums" ist betreff des dort und schon früher von Jay und

1) Dies. Journ. [2] 42, 521. 